\title{
Correlational Origin of the Roton Minimum
}

\author{
G. J. Kalman ${ }^{1}$, P. Hartmann ${ }^{1,2}$, K. I. Golden ${ }^{3}$, A. Filinov ${ }^{4}$ and Z. Donkó ${ }^{1,2}$
}

1 Department of Physics, Boston College - Chestnut Hill, MA 02467, USA

${ }_{2}^{2}$ Research Institute for Solid State Physics and Optics of the Hungarian Academy of Sciences - H-1525 Budapest, $P O B$ 49. Hungary

3 Department of Mathematics and Statistics, Department of Physics University of Vermont, Burlington, Vermont 05401-1455, USA

4. Institut für Theoretische Physik und Astrophysik, Christian-Albrechts-Universität zu Kiel, D-24098 Kiel, Germany

PACS 52.27.Gr - Strongly-coupled plasmas

PACS 67.25.dt - Sound and excitations in ${ }^{4} \mathrm{He}$

\begin{abstract}
We present compelling evidence supporting the conjecture that the origin of the roton in Bose-condensed systems arises from strong correlations between the constituent particles. By studying the two dimensional bosonic dipole systems a paradigm, we find that classical molecular dynamics (MD) simulations provide a faithful representation of the dispersion relation for a lowtemperature quantum system. The MD simulations allow one to examine the effect of coupling strength on the formation of the roton minimum and to demonstrate that it is always generated at a sufficiently high enough coupling. Moreover, the classical images of the roton-roton, rotonmaxon, etc. states also appear in the MD simulation spectra as a consequence of the strong coupling.
\end{abstract}

Since the appearance of the pioneering papers by Landau on the theory of superfluidity [1, the notion of the "roton minimum" in the collective mode dispersion of the system has played a pivotal role in the explanation of the behavior of the superfluid phase of ${ }^{4} \mathrm{He}$. This Letter addresses this old issue from a novel point of view, made possible by results from newly available computer simula$\checkmark$ tion techniques.

The revealing feature of the superfluid behavior is the non-viscous flow of the liquid [2] (or the frictionless mo- tion of an impurity in the liquid 3]) below the critical temperature $T_{c}=2.17 \mathrm{~K}$, and the breakdown of this behavior above a critical flow velocity. In Landau's theory, it is postulated that in order to explain this breakdown, the $\omega(k)$ dispersion curve of the collective excitation must be non-monotonic, starting with a longitudinal $\omega=k s$ acoustic portion, reaching a maximum (the "maxon"), which is then followed by a deep minimum around a $k=k_{\min }$ (the "roton minimum"), such that the $\omega=k s_{\max }$ line, representing the relative motion with respect to the fluid, becomes tangent to the dispersion curve in the neighborhood of the minimum.

Since 1947 the physical origin of the roton minimum and the question whether it $\mathrm{s}$ a part of thelongitudinal phonon eexcitation has been open to conflicting interpretations.
Landau's original suggestion was that the development of the roton minimum had to be sought in the existence of a new collective excitation, quite distinct in nature from the longitudinal acoustic phonon and rather due to a rotationlike collective motion of the fluid: hence, of course, the term by which this phenomenon has become to be known.

Almost a decade later Feynman and Cohen [4 took up the detailed analysis of the ground state of a strongly correlated bose liquid. Their seminal work led to the identification of $S(k)$, the static structure function, as the centrally important quantity in the characterization of the system. Through a series of physical arguments - whose outcome today would be summarized as the "Feynman Ansatz" - Feynman was able to relate the ground state $S(k)$ to the ground state excitation spectrum $\omega(k)$ of the fluid through his famous formula

$$
\omega(k)=\frac{\hbar k^{2}}{2 m S(k)} .
$$

Based on (1), the observation that strong correlations create a sharp maximum in $S(k)$ resulted in the re-emergence of the roton minimum from a rather different physical foundation than that envisioned by Landau. Nevertheless, perhaps surprisingly, the interpretation offered by Feynman still endorsed the association of the roton minimum 
with a vortex-like excitation.

In the subsequent decades while observational data through neutron scattering measurements accumulated and unambiguously revealed the existence of the roton minimum [5] at around $k_{\min }=1.93 \AA^{-1}$ almost as predicted by Landau's hypothesis, the interpretation of its physical origin remained somewhat in a limbo. The idea that the low- $k$ phonon and the high- $k$ roton stem from different sources prevailed: in a scenario developed by Glyde and Griffin in 1990 the roton was viewed as a single particle excitation mixed with the density fluctuation spectrum 6. At the same time, however, in the 1970-s Schneider and co-workers [7] pointed at a different role that the roton minimum seemed to play: namely being the heralded of an incipient liquid-solid phase transition. Still, it took an additional thirty years for the corollary of this statement to be enunciated by Nozieres [8], by suggesting that the physical mechanism responsible for the very creation of the roton minimum is the strong correlations prevailing in the system (although the fact that local minima appear in the dispersion curves of other strongly correlated systems resembling the ${ }^{4} \mathrm{He}$ roton minimum was realized much before that 9]). The purpose of this Letter is to provide evidence, based on recent computer simulations, that favors this latter physical picture.

New developments in condensed matter physics over the past decade, notably the emergence of experimentally available novel 3D and 2D bosonic systems, have created the feasibility of studying the physics of the roton minimum under more controlled conditions. Perhaps the most interesting new system that has appeared along these lines is the two-dimensional liquid of excitonic dipoles. The existence of an excitonic liquid in 2D semiconductor layers and its expected condensation at $T=0$ was predicted by Keldysh and co-workers a long time ago [10]. Subsequently, the possibility of realization of stable excitons in electron-hole bilayers was analyzed by Lozovik and others [1]. It is now fairly well established that with a sufficiently small layer separation $d(d / a<1$, where $a$ is the average interparticle distance within a layer) the description of these excitons by a model of a single 2D layer of point dipoles interacting through a classical dipole-dipole potential is a very good approximation.

Recently, the properties of such a 2D system of bosonic dipoles, both at zero and at finite temperatures have been the subject of intensive analytic and computer simulation efforts. Some experimental observations in GaAs semiconductor bilayers 12 can also be interpreted in terms of this model. Contrasting with liquid ${ }^{4} \mathrm{He}$, the unique feature of the dipole system resides in the simplicity of the interparticle interaction and the possibility of tuning the coupling strength by changing system parameters, such as temperature and density. It is now feasible to trace both the equilibrium behavior and the structure of collective excitations over a wide range coupling strengths and temperatures, by applying various techniques of computer simulation [13,14. This includes the analysis of the generation of the roton minimum and of its evolution as a function of the system parameters. These simulation studies have shed a new light on this old issue. In the following we discuss what can be gleaned from these recent results and point out what conclusion can be drawn from them as to the physical origin of the roton minimum.

The 2D point-dipole system can be described as a collection of $N$ spinless point dipoles, each of mass $m=m_{\mathrm{e}}+$ $m_{\mathrm{h}}$, occupying the large but bounded area $A ; n=N / A$ is the average density. The dipoles are free to move in the $x-y$ plane with dipolar moment oriented in the $z$ direction. The interaction potential is accordingly given by

$$
\varphi(r)=\mu^{2} / r^{3},
$$

where $\mu$ is the electric dipole strength. The coupling strength can be characterized at arbitrary temperature by

$$
\tilde{\Gamma}_{D}=\mu^{2} / a^{3} E_{\mathrm{kin}}
$$

where $E_{\text {kin }}$ is a characteristic kinetic $(a=1 / \sqrt{\pi n}$ is the average inter-particle distance). At zero temperature,

$$
E_{\mathrm{kin}} \simeq \hbar / m a^{2}
$$

and $\tilde{\Gamma}_{D}=r_{D}=r_{0} / a, r_{0}=m \mu^{2} / \hbar^{2}$ is the appropriate measure of the coupling strength; the characteristic length $r_{0}$ is the dipole equivalent of the Bohr radius. In fact, (4) is valid up to an arbitrary factor $\mathrm{O}(1)$ : its exact evaluation would require information about the ground state momentum distribution. In the high-temperature classical domain, with

$$
E_{\mathrm{kin}}=1 / \beta,
$$

$\left(\beta^{-1}=k_{\mathrm{B}} T\right.$ is the thermal energy) and (3) becomes $\Gamma_{D}=\beta \mu^{2} / a^{3}$, in analogy with the $\Gamma=\beta e^{2} / a$ coupling parameter for classical Coulomb liquids. The comparison of the two limits of $\tilde{\Gamma}_{D}$ allows one to establish the correspondence between the high temperature classical) and low temperature (quantum) domains through the equivalence $\Gamma_{D} \Leftrightarrow r_{D}$.

The behavior of the dipole system can be analyzed over a wide range of temperatures and densities. The temperature domains may be identified through the value of the degeneracy parameter

$$
\Theta=\beta \hbar^{2} / 2 m a^{2} .
$$

As the quantum, $\Theta>1$ and classical, $\Theta<1$ and regimes. The equilibrium in the quantum domain exhibits a great richness of phenomena, such as condensation and quasi-condensation, superfluidity and crystallization, whose competition leads to an intricate low temperature phase diagram [15], quite at variance with the behavior in the classical domain. However, as far as the collective excitation spectrum is concerned, the situation is different: collective modes of many-body systems governed by the cooperative motion of many particles seem to be robustly insensitive to statistics (an obvious example is the 
plasmon in charged particle systems, which exhibits very much the same features in classical plasmas as in the degenerate electron gas).

We proceed now to compare the description of the collective mode dispersion based on two sets of computer simulation data. To the first set belong data pertaining to the static equilibrium properties of low temperature quantum dipole systems, through zero temperature quantum Monte Carlo (QMC) 13 and low-temperature path integral Monte Carlo (PIMC) 15. simulations. The second set of data originates from a Molecular Dynamics (MD) simulation of the dynamics of a classical point dipole system. The QMC simulation of the zero temperature quantum dipole system in the strong coupling regime has been pursued by Astrakharchik et al. [13] and by Mazzanti et al. 16. In these works static structure factors have been generated as functions of the zero temperature coupling parameter $r_{D}$. The dispersion relation for the longitudinal dipole oscillations was obtained through two analytic approximations: first, through the Feynman formula (11), and, second, by incorporating the Feynman frequencies in a more sophisticated formalism based on the correlated basis function (CBF) approximation (extensively reviewed by Feenberg [17, Campbell 18, and Woo 19]), which takes account of the effect of the three-phonon interaction term in the Hamiltonian. In the PIMC simulation approach [20] followed by Filinov and co-workers [15], similar data for low but finite temperatures have been generated. Coming now to the second set of data, a program of MD simulation of the dynamics of a classical point dipole system has been pursued by the authors, along the pattern used for simulating other strongly interacting classical systems 21. In this approach the dynamical structure function $S(k, \omega)$ is obtained for a range of values of the classical coupling parameter 22] $\Gamma_{D}$ whose peaks reveal the collective excitation spectrum of the system. It should be emphasized that, in contrast to the work of Astrakarchik et. al. 13, this latter method provides direct information without the intervention of any further analytic approximation on the dispersion relation for the longitudinal dipole oscillations in the physical, albeit classical, system. Within the presently available computational technology it would be of course futile to expect data of the same kind to be created for quantum systems, where, to date, one has to rely on the intermediary of approximation techniques to connect the dynamics of the system with the available equilibrium information.

We now compare the results from the two sets of data, which are presented in Fig. 11 for approximately equivalent values of the classical and zero temperature coupling parameters $\tilde{\Gamma}_{D}$. The main statement of this paper derives from a number of conclusions that can be drawn from the inspection and analysis of these graphs.

1. There is no major difference in the morphologies of the dispersion curves pertaining to the quantum and classical systems; in particular, both of them show

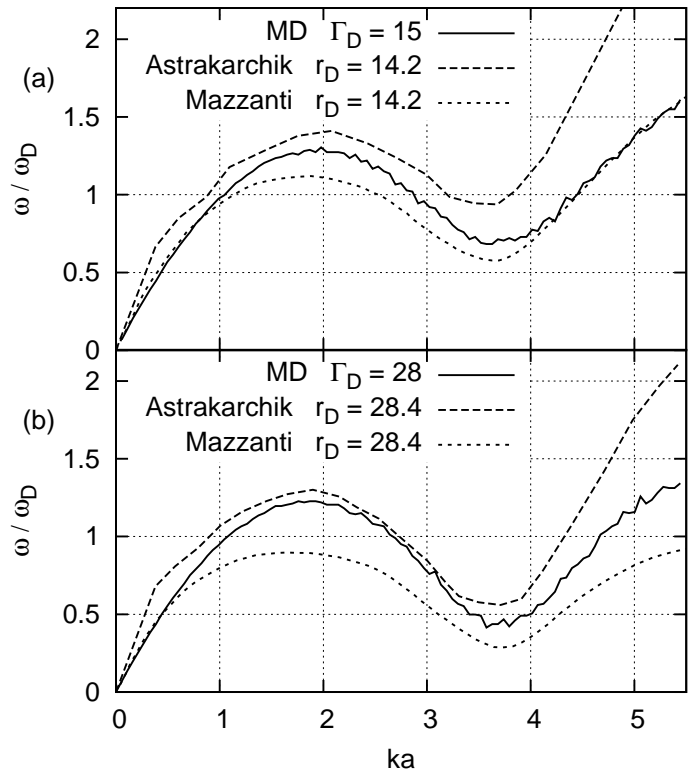

Fig. 1: Comparison of dispersion curves: MD (classical molecular dynamics), Astrakarchik 13] (Feynman upper bound based on QMC), Mazzanti [16] (presumed lower bound). (a) $\Gamma_{D}=$ 15 , (b) $\Gamma_{D}=30$.

the formation of the roton minimum in the vicinity of $k a \simeq 3.5$.

2. Whether the roton minimum develops in the dispersion curve depends solely on the coupling parameter $\tilde{\Gamma}_{D}$ : the minimum value for the curve to become nonmonotonic is around $\tilde{\Gamma}_{D}=5$, both for the classical and quantum system.

3. With increasing value of $\tilde{\Gamma}_{D}$, the roton minimum becomes deeper, reaching an absolute minimum. For the $T=0$ curve this occurs at $\tilde{\Gamma}_{D}=28.6$, near the crystallization value [13] $\tilde{\Gamma}_{D}^{*}=30.2$.

4. The classical dispersion curve is sandwiched between the quantum Feynman and the quantum CBF curves; its being positioned below the Feynman curve is expected, since the latter constitutes an upper bound only. As to the CBF construction, it is difficult to assess its accuracy by analytic means; however, CBF calculations performed for ${ }^{4} \mathrm{He}$ with model potentials have agreed well with measured spectra, with deviations attributable to the uncertainty of the actual interaction potential 23. Thus, one is probably on safe grounds by accepting the CBF curve as a lower bound.

All these observations converge now towards a coherent physical picture that clearly suggests that the roton minimum is the consequence of strong correlations, which are basically a classical phenomenon, in the system. Quantum dynamics, Bose statistics, condensation, superfluidity, etc. seem to have very little influence either on the formation 


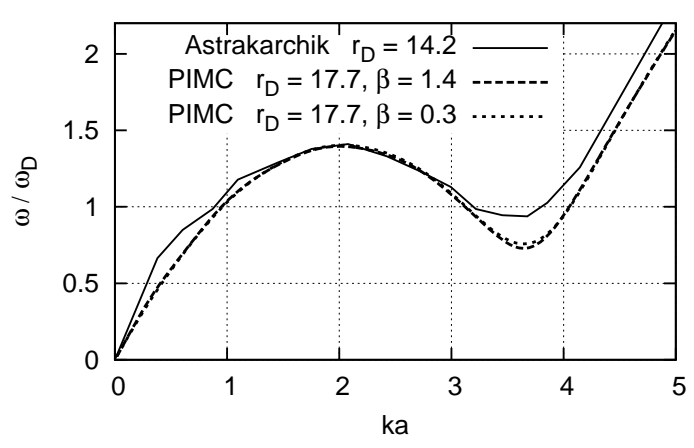

Fig. 2: Comparison of $T=0$ and finite low temperature dispersions. Note that the finite temperature modifications are very small.

or on the structure of the roton minimum. It would be a strange coincidence indeed, if hybridization with vortexlike or other unidentified excitations were responsible for the creation of the same phonon dispersion characteristics in bose liquids as they are manifested through strong correlations in the equivalent classical systems.

Having made our central observation, there are a few more issues to be addressed. Our discussion on the quantum system so far has been limited to the $T=0$ ground state, whose analysis is sufficient to establish the correlational origin of the roton minimum. Finite temperatures are, however, now available: the recent PIMC calculations [15] have generated $S(k)$ data in the $0.5<\Theta<3.3$ domain (on both sides of the superfluid/normal fluid transition point). The generalization of the zero temperature Feynman formula (11) to finite temperatures becomes

$$
\omega(k) \tanh \left[\frac{\beta \hbar \omega(k)}{2}\right]=\frac{\hbar k^{2}}{2 m S(k)} .
$$

The PIMC dispersion curves based on (7) for three representative $\Theta$ values, together with the QMC ground state dispersion are displayed in Fig. 2, The difference between the $T=0$ and $T \neq 0$ dispersions is strikingly small (except near $k=0$, a region for which the inaccuracy of the QMC simulations 13] has already been pointed out 24, further corroborating the expectation that as far as the dispersion properties of the collective excitations are concerned, the transition from the $T=0$ degenerate to the $T \rightarrow \infty$ classical phase is smooth and eventless.

Quite at variance with the dispersion, one expects that the lifetime of the excitations (i.e. the damping of the oscillations) is strongly affected by rising temperature. Here one has to rely on what is known from observations on liquid ${ }^{4} \mathrm{He}$, which then can be compared with relevant information gleaned from the classical MD simulations. From the analysis of $S(k, \omega)$ with the aid of neutron scattering experiments 25] we know that the character of the roton minimum peak undergoes a dramatic change as the system passes from the superfluid to the normal phase: while it is very sharp in the superfluid phase, it becomes broad in the latter and as the temperature continues to rise above its

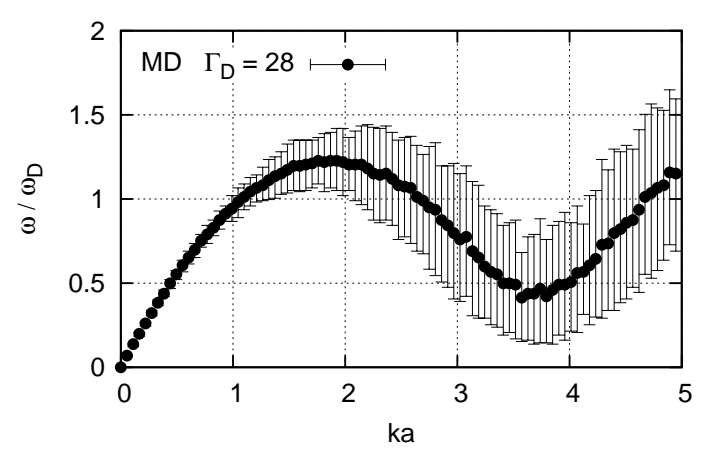

Fig. 3: Broadening of the phonon-maxon-roton line due to temperature effect. Error bars indicate the width (full width at half maximum) of the spectral peak.

critical value it becomes quite ill-defined. Thus one would expect a similarly broadened roton peak in the classical MD generated $S(k, \omega)$. This indeed is the case. An examination of Fig. 3 that portrays $S(k, \omega)$ and the width of the roton peak for a sequence of increasing $\Gamma_{D}$ values shows that the width decreases for higher coupling, yet remains considerable up to crystallization. Beyond observing the qualitatively identical behavior in the classical $2 \mathrm{D}$ dipole system and ${ }^{4} \mathrm{He}$, quantitative comparison would be quite meaningless, first because of the entirely different interaction potentials in the two systems, and, second, because studying the effect of the variation of the coupling strength is obviously not an available option in $4 \mathrm{He}$ experiments. The conclusion to be drawn from the comparison is, however, that the temperature engendered damping of the rotons is similar in the classical and quantum systems and its occurrence in no way invalidates the link between the finite $T$ classical and the $T \simeq 0$ quantum systems.

If we now accept the premise that the roton minimum experienced in the bose systems studied are caused by the strong correlations of classical origin, one may wonder about the generality of the link between strong correlations and roton-like behavior for other many- body systems. First, would systems governed by interaction potentials other than the ones that have been investigated exhibit a similar behavior? With a high level of confidence one can predict that this would indeed be the case. In fact, it was prior to the MD analysis of the dipole system that the classical "roton minimum" phenomenon was established for Coulomb and Yukawa systems [21]. There is no reason to believe that the mapping of this behavior to the equivalent low temperature Bose system, should such a system become available for observation, would not occur in the same fashion as it does for a system of dipoles. Second, could the classical roton minimum behavior be mapped to a strongly interacting low temperature system of fermions in the same way as it is to a system of bosons? We do not have enough observational or theoretical understanding of such systems to provide a definite answer. Nevertheless, it is clear that the fundamentally different low temperature behaviors of the fermi and bose systems 
would make a similarity unlikely. The issue is that in a bose condensate or quasi-condensate the single particle excitations are suppressed, which makes the unimpeded development of the collective excitation, even in the high$k$ domain where the roton minimum is to occur, possible. There is no such suppression in the fermi system. Taking the electron liquid as a paradigm, the high- $k$ development of the plasmon branch is profoundly affected by the presence of the electron-hole pair continuum. Based on a simplified model [26, with a measure of caveat it can be argued that the pair (and multi-pair) continuum not only generates a strong damping to the collective mode, but also inhibits its evolution in the high- $k$ domain. Thus it seems safe to conclude that the condensed (or quasicondensed) bose system is the sole candidate for the clear manifestation of the correlation induced roton minimum.

The final issue we want to address is what has become to be known in the ${ }^{4} \mathrm{He}$ studies as the issue of the tworoton state. It was shown first by Cowley and Woods [27] that in addition to the phonon excitation, a high frequency branch can be identified in the observed mode spectrum of the liquid ${ }^{4} \mathrm{He}$ : this mode was identified as a two roton excitation, with a frequency in the vicinity of twice the frequency of the roton minimum and rather insensitive to the wave number. More recent neutron scattering studies 28 have found additional branches with higher frequencies that can be attributed to roton-maxon and maxonmaxon excitations.

The identification of these high frequency states in terms of the excitation of two quasi-particles goes back to the classic paper of Miller, Pines and Nozieres 29. In the original model, the quasi-particles were non-interacting: the effect of the interaction between them was suggested by Pitaevski 30 and first considered in detail in the pioneering series of works by Ruvalds, Zawadowski, Bedell , Pines and collaborators [30. In these works, depending on the sign of the interaction (attractive vs. repulsive), a downward or upward shift from the simple additive nominal values of the high frequency excitations is predicted.

The generation of higher harmonics and combination frequencies is a well-known nonlinear effect [31, due to the inherent nonlinearity of the interaction between the constituent $\left({ }^{4} \mathrm{He}\right.$, dipoles e.g.) particles. Given a dispersion curve, any pair of frequencies along the curve is a candidate for the generation of combination frequencies: it is, however, the frequencies in the vicinity of extrema of the dispersion curve, where the density of states is the highest, which are primarily responsible for creating observable new branches of collective excitations. In this paper we have argued that the generation of the roton minimum and of the maxon maximum in strongly coupled bose condensate (quasi-condensate) liquids is a universal effect due the strong interaction between their constituents. A corollary to this statement is now that the roton- roton, roton-maxon and maxon-maxon states should also be identifiable for the equivalent classical liquid through the appearance of a significant weight in the density correla-

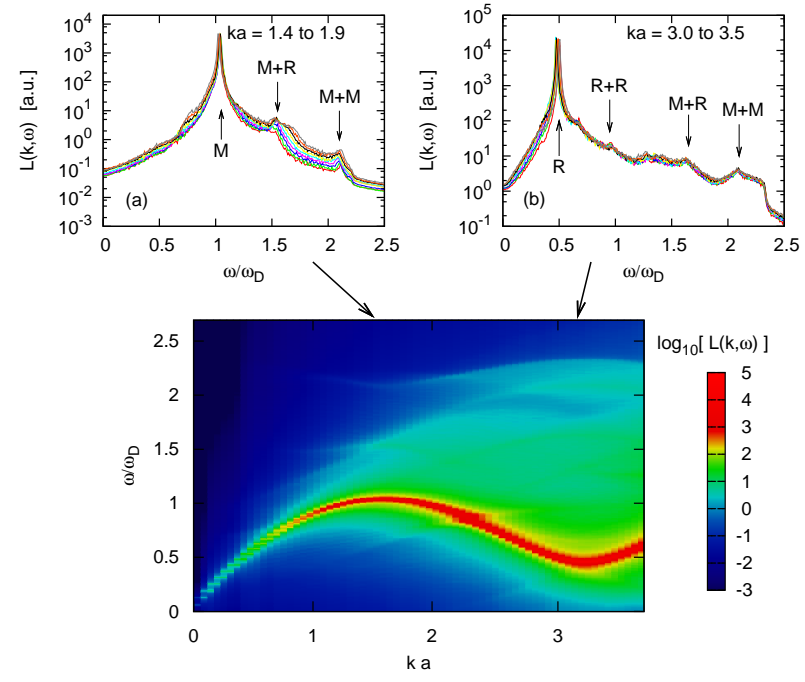

Fig. 4: (color online) Illustration of the appearance of the $\mathrm{R}+\mathrm{R}, \mathrm{R}+\mathrm{M}, \mathrm{M}+\mathrm{M}$ combination frequencies in the longitudinal current-current correlation functions $L(k, \omega)$, measured along the direction of the nearest neighbor in a hexagonal lattice). The center color map of the $L(k, \omega)$ fluctuation spectra shows the strong primary dispersion and the "ghosts" of the combination frequencies. The small panels show vertical cross-sections taken for the selected group of wave-numbers, where the appearance of the combination frequencies is the most manifest.

tion spectra at the corresponding combination frequencies.

The MD analysis of $S(k, \omega)$ of the strongly coupled dipole liquid when extended into the high frequency regime up to and beyond twice the maxon frequency indeed reveals these expected features. In Fig. 4 we have displayed a series of $S(k, \omega)$ spectra for a wide range of $\mathrm{k}$ values. In order to emulate the low level of the background noise characteristic of the superfluid, the graphs were taken at $\Gamma_{D}=500$, well beyond the crystallization value, but at a value of coupling where the thermal motion is of sufficient amplitude to generate nonlinear effects. Thus these graphs may be considered appropriate to illustrate a trend, but a detailed analysis of the features exhibited in them is not our purpose here: it is sufficient to point out that the essential feature, namely the accumulation of weight in the vicinity of three combination frequencies, the roton-roton $(\mathrm{R}+\mathrm{R})$, roton-maxon $(\mathrm{R}+\mathrm{M})$, and maxonmaxon $(\mathrm{M}+\mathrm{M})$ is clearly visible. From other works we know that the generation of harmonics, similarly to the development of the roton minimum itself, appears to be a general feature of strongly coupled liquids with Yukawa, Coulomb and other similar types of interaction 32 as well. The relative amplitudes of these harmonics are very much functions of the particular type of interaction potential prevailing in the system. Thus, attempts at a quantitative comparison with experimental data on liquid ${ }^{4} \mathrm{He}$ would in all likelihood not be useful. On the other hand, the positions of the combination frequencies, i. e. their shifts from their nominal values, is due, in the language of quasipar- 
ticle states, to the interaction between the quasiparticles and, as such, their comparison with the predictions of the RZ theory as applied to the 2DDS would certainly be of interest.

To summarize, in this paper we have provided compelling support for two earlier suggestions [7, 8, that the formation of maxon-roton excitations in condensed or quasi-condensed bose systems is the result of a basically classical strong correlational effect. Our assessment is based on the analysis of the collective mode dispersion of the strongly coupled 2D bosonic dipole system (2DDS) below and above the superfluid transition temperature 13, 14. This dispersion exhibits a phonon-maxon-roton structure, very much the same as the collective mode dispersion in ${ }^{4} \mathrm{He}$. Paralleling these studies, we investigated 22 the dispersion of the strongly coupled 2DDS in the high-temperature classical domain through molecular dynamics (MD) simulations: we have discovered a remarkable congruence between the collective mode dispersions of the classical and quantum systems, pointing at a common origin of these phenomena, the shaping of the longitudinal phonon dispersion by strong interparticle correlations. Models that seek the origin of the roton in excitations different in nature from the phonon seem to be incompatible with this evidence. Moreover, the MD simulations now can be trusted to provide a first-time glimpse into the actual behavior of the dynamics of the strongly correlated system without reliance on the intermediary of models. The approach that describes correlational effects in the dynamical behavior through the static structure function is superseded by the direct dynamical MD simulations. Furthermore, our observations of a considerable weight in the MD density fluctuation spectrum in the vicinity of the roton-roton, roton-maxon, and maxon-maxon combination frequencies, similar to what has been observed in neutron scattering experiments [28] in ${ }^{4} \mathrm{He}$, may be regarded as additional corroboration of the underlying strong correlational classical model.

We wish to acknowledge useful discussions with A. Zawadowski. This work has been partially supported by OTKA-PD-75113, OTKA- K-77653, MTA-NSF/102, NSF Grants PHY-0813153, PHY-0812956 and PHY-0903808; it was also supported by the Janos Bolyai Research Grant of the Hungarian Academy of Sciences.

\section{REFERENCES}

[1] Landau L., J. Phys. U.S.S.R., 5 (1941) 71; J. Phys. U.S.S.R., 11 (1947) 91; Phys. Rev., 75 (1949) 884.

[2] Kapitza P., Nature, 141 (1938) 74; Allen J. F. and Misener A. D., Nature, 141 (1938) 75; Griffin A., Excitations in a Bose-Condensed Liquid (University Press) 1993.

[3] Allum D. R. et Al, Phys. Rev. Lett., 36 (1976) 1313.
[4] Feynman R. P., Phys. Rev., 94 (1954) 262; in: Progress in Low Temperature Physics vol. 1, ch. 2, edited by C. G. Gorter (North Holland) 1955; Feynman R. P. and Cohen M., Phys. Rev., 102 (1956) 1189; Cohen M. and Feynman R. P., Phys. Rev., 107 (1957) 13.

[5] Woods A. D. B., Phys. Rev. Lett., 14 (1965) 355; CowLEY R. A. and Woods A. D. B., Can. J. Phys., 49 (1971) 117; Andersen K. H. et Al., J. Phys. Cond Matter., 6 (1994) 821; FAK B. and Andersen K. H., Phys. Lett. A, 160 (1991) 468; Stirling W. G., in: Excitations in Two-Dimensional and Three-Dimensional Quantum Flu$i d s$ - NATO ASI 25\%, p. 25, edited by WYATT A. F. D. and Lauter H. J. (Plenum Press, NY) 1990.

[6] Glyde H. R. and Griffin A., Phys. Rev. Lett., 65 (1990) 1454.

[7] Schneider T. and Enz C. P., Phys. Rev. Lett., 27 (1971) 1186; Schneider T., Srinivasan G., and Enz C. P., Phys. Rev. A, 3 (1972) 1528.

[8] Nozieres Ph., J. Low Temp. Phys., 137 (2004) 45.

[9] e. g. Stirling W. G., in: Excitations in TwoDimensional and Three-Dimensional Quantum Fluids NATO ASI 25\%, edited by WyatT A. F. D. and Lauter H. J. (Plenum Press, NY) 1990.

[10] Keldysh L. V. and Kopaev Yu. V., Sov. Phys. Solid State, 6 (1964) 2791; Kelydsh L. V. and Kozlov A. N., Zh. Eksp. Teor. Fiz., 54 (1968) 978, Sov. Phys. JETP, 27 (1968) 521.

[11] Lozovik Yu. E. and Berman O. L., Sov. Phys. JETP, 84 (1997) 1027; Phys. Solid State, 40 (1998) 1228; J. Phys.:Condens. Matter, 14 (2002) 12457; DE PALO S., Rapisarda F., and Senatore G., Phys. Rev. Lett., 88 (2002) 206401; Hartmann P., Donkó Z., and Kalman G. J., Europhys. Lett., 72 (2005) 396; RANGANATHAN S. and Johnson R. E., Phys. Rev. B, 75 (2007) 155314.

[12] Fluegel B., Mascarenhas A., Snoke D. W., PfeifFER L. N., and West K., Nature Photonics, 1 (2007) 701.

[13] Astrakarchik G. E., Boronat J., Kurbakov I. L., and Lozovik Yu. E., Phys. Rev. Lett., 98 (2007) 060405.

[14] Lozovik Yu. E., Kurbakov I. L., Astrakharchik G. E., Boronat J., and Willander M., Solid State Commun., 144 (2007) 399; Astrakharchik G. E., Boronat J., Casulleras J., Kurbakov I. L., and Lozovik Yu. E., Phys. Rev. A, 75 (2007) 063630; Filinov A. V., Ludwig P., Lozovik Yu. E., Bonitz M., and Stolz H., J. Phys.: Conf. Ser., 35 (2006) 197; Filinov A. V., LudwiG P., Golubnychyi V., Bonitz M., and Lozovik Yu. E., Phys. Stat. Sol. (c), 0 (2003) 1518; Kalman G. J., Golden K. I., Filinov A. V., Hartmann P., Bonitz M., and Donkó Z., Collective mode dispersion in a twodimensional dipole system, 13th International Conference on Physics of Non-ideal Plasmas, Chernogolovka, Russia, September 2009.

[15] Filinov A. V., Ludwig P., Bonitz M., and LozoviK Yu. E., J. Phys. A: Math. Theor., 42 (2009) 214016.

[16] Mazzanti F., Zillich R. E., Astrakharchik G. E., and Boronat J., Phys. Rev. Lett., 102 (110405).

[17] Feenberg E., Theory of Quantum Fluids (Academic Press, New York) 1967 and 1969.

[18] Campbell C. E., in: Progress in Liquid Physics, edited by Croxton C. A. (Wiley, New York) 1978.

[19] Woo C. W., in: The Physics of Liquid and Solid Helium 
vol. I, edited by Ketterson J. B. and Bennemann K. H. (Wiley, New York) 1976.

[20] Introduction to Computational Methods in Many Body Physics, edited by Bonitz M. and Semkat D. (Rinton Press, Princeton) 2006.

[21] Donkó Z., Kalman G. J., Hartmann P., J Phys. Cond. Matter, 20 (2008) 413101.

[22] Golden K. I., Kalman G. J., Donkó Z., and HartMANn P., J. Phys. A: Math. and Theor., 42 (2009) 214017.

[23] Manousakis E. and Pandharipande V. R., Phys. Rev. B, 30 (1984) 5064; Phys. Rev. B, 33 (1986) 150.

[24] Golden K. I., Kalman G. J., Donko Z., and Hartmann P., Phys. Rev. B, 78 (2008) 045304; Phys. Rev. B, 78 (2008) 239905(E).

[25] Andersen K. H. et Al., J. Phys. Cond. Matter., 6 (1994) 821 and references therein.

[26] Golden K. I., Mahassen H., Kalman G. J., Phys. Rev. E, 70 (2004) 026406.

[27] Cowley R. A. and Woods A. D. B., Can. J. Phys., 49 (1971) 177.

[28] Juge K. J. and Griffin A., J. Low Temp. Phys., 97 (1994) 105 and references therein.

[29] Miller A., Pines D., and Nozieres P., Phys. Rev., 127 (1962) 1452.

[30] Pitaevskin L. P., Zh. Eksp. Teor. Fiz., 36 (1958) 1169; Sov. Phys. JETP, 9 (1959) 830; Ruvalds J. and ZawADowski A., Phys. Rev. Lett., 25 (1970) 333; Zawadowski A., Ruvalds J., and Solana J., Phys. Rev. A, 5 (1972) 399; Bedell K., Pines D., and Zawadowski A., Phys. Rev. B, 29 (1984) 102.

[31] Weiland J. and Wilhelmsson H., Coherent nonlinear interaction of waves in plasmas (Pergamon Press, Oxford and New York) 1977; Ambegaokar V. ET AL., in: Lattice Dynamics, p. 261, edited by Wallis R. F. 1065.

[32] Hartmann P., Donkó Z., Tierney K. P., Lee C. J., and Kalman G. J., J. Phys. A: Math. Theor., 42 (2009) 214040 . 